\title{
A comparison of Bayesian and Fourier methods for frequency determination in asteroseismology
}

\author{
T. R. White ${ }^{1}$, B. J. Brewer ${ }^{1,2}$, T. R. Bedding ${ }^{1}$, D. Stello ${ }^{1}$ and H. Kjeldsen ${ }^{3}$ \\ ${ }^{1}$ Sydney Institute for Astronomy (SIfA), School of Physics, University of Sydney, \\ NSW 2006, Australia \\ 2 Department of Physics, University of California, Santa Barbara, CA 93106-9530, \\ USA \\ ${ }^{3}$ Danish AsteroSeismology Centre (DASC), Department of Physics and Astronomy, \\ Aarhus University, DK-8000 Aarhus C, Denmark
}

\begin{abstract}
Bayesian methods are becoming more widely used in asteroseismic analysis. In particular, they are being used to determine oscillation frequencies, which are also commonly found by Fourier analysis. It is important to establish whether the Bayesian methods provide an improvement on Fourier methods. We compare, using simulated data, the standard iterative sine-wave fitting method against a Markov Chain Monte Carlo (MCMC) code that has been introduced to infer purely the frequencies of oscillation modes (Brewer et al. 2007). A uniform prior probability distribution function is used for the MCMC method. We find the methods do equally well at determining the correct oscillation frequencies, although the Bayesian method is able to highlight the possibility of a misidentification due to aliasing, which can be useful. In general, we suggest that the least computationally intensive method is preferable.
\end{abstract}

Accepted: June 7, 2010

\section{Introduction}

Bayesian methods are increasingly being used for asteroseismic analysis. Most effort has been directed at extracting mode parameters by fitting to the Fourier power spectrum (e.g. Appourchaux 2008; Benomar 2008; Benomar et al. 2009; Gaulme et al. 2009), but there have also been applications that involved fitting directly to the time series (Brewer et al. 2007; Brewer \& Stello 2009). It is the 
latter approach, which can be thought of as an alternative to calculating the power spectrum, that is the subject of this paper. Determining the frequencies at which stars oscillate is fundamental to asteroseismology. The first step in doing so is generally to calculate the power spectrum of the time series, in which the frequencies of oscillation will appear as peaks. However, this is complicated by noise and aliasing so that it is not always immediately obvious which peaks are real. In this paper, we use simulated data to determine if a Bayesian Markov Chain Monte Carlo code is more effective at determining real frequencies than a standard iterative sine-wave fitting code.

\subsection{Iterative sine-wave fitting}

To systematically extract the peaks that are most likely to be real, an iterative algorithm (Roberts et al. 1987) is commonly used (e.g. Carrier \& Bourban 2003; Kjeldsen et al. 2005; Bedding et al. 2007). This is referred to as iterative sine-wave fitting (also called CLEAN). In this algorithm, the discrete Fourier transform of the time series is calculated, generating the amplitude spectrum. The highest peak in the amplitude spectrum is identified and the corresponding sinusoid is then subtracted from the time series. The discrete Fourier transform of the residual time series is then calculated, and the process is repeated until the amplitude of the highest peak is below a chosen threshold. The result is a list of frequencies, amplitudes and phases that account for most of the variations in the time series.

However, this technique may run into difficulties. It is possible for alias peaks (sidelobes) to be enhanced by noise to the point where they have a higher amplitude in the Fourier spectrum than the genuine peak. In this case, iterative sine-wave fitting programs will identify the alias and subtract it from the time series. The amplitude of the genuine peak in the Fourier spectrum will be diminished by this process to the point where it may not be found at all. This problem may be exacerbated if the separation between oscillation peaks is approximately an integer multiple of the typical period between gaps in the data (usually one cycle per day in ground-based data) since the alias peaks may reinforce. In addition to this, noise peaks may obscure the signal.

Other concerns extend from the nature of Fourier methods. Bretthorst (1988) has shown that the power spectrum is an optimal procedure only in the case where only one mode is present, or if multiple, they are well separated in frequency. In practice, particularly with solar-like oscillations, this is rarely the case, with multiple modes often closely spaced in frequency. A further consideration is that the oscillation modes will vary in time such that a single mode must be represented by multiple sinusoids in the Fourier series. An additional problem can arise from the nature of the algorithm itself: when a frequency is 
found, and its amplitude and phase fitted to the time series, the corresponding sinusoid is subtracted. However as these parameters cannot be exact, biases are introduced into the residual time series.

\subsection{Bayesian Methods}

The above concerns have led to the consideration of Bayesian methods for determining frequencies. Probability theory may be used as a mathematical model of our belief in the plausibility of various hypotheses. Our knowledge of a set of parameters, $\theta$, given prior information and assumptions, $I$, is represented by the prior probability distribution, $p(\theta \mid I)$. Given new data, $D$, Bayes' theorem,

$$
p(\theta \mid D, I) \propto p(\theta \mid I) p(D \mid \theta, I)
$$

tells us about our new state of knowledge, the posterior distribution. The distribution $p(D \mid \theta, I)$, the probability distribution for the data given the parameters, is a measure of how well the data are predicted by the model. In the case where the data are known and fixed, $p(D \mid \theta, I)$ becomes dependent on $\theta$ only and is called the likelihood function. How much we believe our model depends on both how well we originally believed it (the prior distribution), and on how well it predicts the new data. It is important to note that probabilities are always conditional on the underlying background information and/or assumptions $I$, even when these are not explicitly stated. Bayes' theorem provides a means of finding the most probable model that could produce the observed data.

A method using this probabilistic reasoning has been developed by Brewer et al. (2007) and applied to the subgiant stars $\nu$ Indi (Bedding et al. 2006; Carrier et al. 2007) and $\beta$ Hydri (Bedding et al. 2007). This method utilises a version of the Metropolis-Hastings algorithm (Neal 1993), itself a Markov Chain Monte Carlo (MCMC) algorithm (Gregory 2005), to determine the most likely set of frequencies (represented by $\theta$ above) that could give rise to observed data. Essentially, the code samples the $\theta$ parameter space - the number of frequencies and their values - through a random walk with more time being spent in regions of higher probability. At each iteration of the code, the current state is randomly perturbed. If the perturbation results in a higher posterior probability then it is accepted as the new state, otherwise the perturbed state is accepted with a probability proportional to the ratio of the new posterior probability density to the old.

The method of Brewer et al. (2007) analyses the observed time series directly. To do this it finds the most likely sinusoids that fit the time series, assuming that the time series is composed of a small number of sinusoidal signals (one per mode) and Gaussian noise. This assumption is not generally valid due to the stochastic nature of excitation and damping of the oscillations. 
Some methods that take this in to account are able to infer frequencies and line widths by fitting to the power spectrum (e.g. Appourchaux 2008). However, the presence of gaps in the data, and the stochastic nature of oscillations results in the possibility that information will be lost in the power spectrum (Bretthorst 1988). It was this potential loss of information from using the power spectrum that was one of the reasons for considering Bayesian methods in the first place. The convenience of the power spectrum cannot be doubted and for data with good coverage and long mode lifetimes, the loss of information is negligible. Nevertheless it would be ideal to have a method that both analyses the time series directly and takes into account that the oscillations are not purely sinusoidal. Such a method has been developed (Brewer \& Stello 2009), but is unfortunately computationally intensive and is currently only practical for short time series (fewer than $\sim 1500$ points). For this reason, it is the method of Brewer et al. (2007) we use in our comparison here with Fourier methods.

A major (although potentially dangerous) feature of Bayesian inference is the ability to incorporate extra knowledge in determining which parameters are more likely by choosing a descriptive prior distribution. For some stellar oscillations, namely high-overtone, low-degree acoustic oscillations in spherically symmetric stars, modes are usually expected to follow the asymptotic relation,

$$
\nu_{n, I}=\Delta \nu\left(n+\frac{1}{2} I+\epsilon\right)-I(I+1) D_{0},
$$

where $\Delta \nu$ is called the large separation and depends on the sound travel time across the whole star, $D_{0}$ is sensitive to the sound speed near the core and $\epsilon$ is sensitive to the surface layers (Tassoul 1980, 1990). With this in mind, a prior distribution could be chosen in which a regular spacing of modes is anticipated. The MCMC code would then favour finding peaks with a regular spacing over finding peaks that do not fit this pattern. This was a feature of the code when it was introduced by Brewer et al. (2007) and tested on simulated data (which did have a constant separation between modes) and when it was used on real observations of $\nu$ Indi by Bedding et al. (2006) to find the large separation. The value inferred by the Bayesian method for the large separation, $\Delta \nu=24.25 \pm 0.25 \mu \mathrm{Hz}$, agreed well with the value obtained from the peak of the autocorrelation function of the power spectrum, a Fourier method.

As mentioned, this use of a descriptive prior distribution can be dangerous. Although the modes of oscillation will be separated in frequency by approximately equal amounts, in general there will be a departure from the asymptotic relation. The large separation itself may be frequency-dependent. By using a prior that is too prescriptive, this may be missed by the Bayesian method. To avoid this possibility, the method described by Brewer et al. (2007) incorporated a uniform component and a regular pattern of Gaussian peaks, as opposed to delta functions. However, this may still be too prescriptive. A further analysis 
of the data on $\nu$ Indi showed that the large separation was indeed a function of frequency, with the average large separation of $\Delta \nu=25.14 \pm 0.14 \mu \mathrm{Hz}$ larger than first realised (Carrier et al. 2007).

Further use of this method was made by Bedding et al. (2007) on $\beta \mathrm{Hy}$ dri. They used the Bayesian method as a supplement to traditional Fourier methods to determine individual frequencies, although no assumptions about the frequency distribution were made. To avoid detecting noise peaks in the Fourier analysis, only the peaks with a signal-to-noise ratio $(\mathrm{S} / \mathrm{N}) \geq 4$ were included. Comparing the results with the Bayesian method, one extra peak was found by the Bayesian method, lying precisely on the $I=1$ ridge of the échelle diagram. The same peak was found by the Fourier iterative sine-wave fitting with $\mathrm{S} / \mathrm{N}=3.0$.

These studies of $\nu$ Indi and $\beta$ Hydri have obtained similar results using both Bayesian and traditional Fourier method. The question arises as to which method, if either, is superior. Brewer et al. (2007) suggested that the Bayesian method was less susceptible to aliasing and, by effectively fitting multiple sinusoids simultaneously, was less susceptible to any issues caused by the subtraction of modes from the time series. On the other hand, the Bayesian method is more computationally intensive for large data sets, and it has been suggested that its advantage over the traditional Fourier methods is with shorter, noisier, and more incomplete data sets. Despite these presumed advantages, a detailed comparison of the methods has not yet been made. It is the purpose of this paper to do this comparison on simulated data for which the frequencies are known.

\section{Programs being tested}

Two different iterative sine-wave fitting programs were used, both written by one of us (HK). They differ in that one program re-adjusts the frequencies, amplitudes and phases of all previously extracted peaks at each iteration, in an attempt to improve the fit. However, there is a time penalty to this, and it may not necessarily lead to improved results because the process may be unstable. Hereafter, the version which does not recalculate parameters at each iteration is referred to as fast and the other as slow.

The program used to evaluate Bayesian methods of determining frequencies was written by one of us (BJB) and is a variation of the program introduced by Brewer et al. (2007), which implements a Markov Chain Monte Carlo (MCMC) algorithm. The principles of the original program have been discussed above. Here, we outline how our implementation of the code differs.

The most important difference is the choice of the prior probability distribution. Our choice here can have a significant impact on the results obtained. 
Brewer et al. (2007) used a prior distribution which took into account that the frequencies of low-degree $p$-mode oscillations are expected to be approximately given by Equation 2. This is not a valid assumption if a mode exhibits mixed $p$ - and $g$-mode behaviour, as in evolved stars, or if the large separation, $\Delta \nu$, is not constant with frequency. Setting this prior will have a significant impact on the posterior distribution, potentially placing unreasonably high confidence in low signal-to-noise peaks that fit a regular spacing, and down-weighting real peaks that do not. For our implementation of this method, we have chosen only to use a uniform prior that does not anticipate the separation of peaks to avoid the possibility of the code finding a regular spacing that may not be there. The uniform prior is given by

$$
g=\frac{1}{\nu_{\max }-\nu_{\min }},
$$

where $\left(\nu_{\min }, \nu_{\max }\right)$ is the range of frequencies over which we attempt to find oscillations.

Since Brewer et al. (2007) used a sharply peaked prior, it was possible for the program to become stuck in a local minimum and not adequately sample the parameter space. To overcome this problem parallel tempering was used with respect to the prior. In our case we do not use parallel tempering since the uniform prior is without the sharp peaks that could cause the chains to become stuck in local minima.

Will this change to the prior have much impact on the number of frequencies used in the models at each iteration? That is, how effective was the use of a prior that expected a regular spacing of peaks at suppressing peaks that did not meet this criteria? Figure 3 of Brewer et al. (2007) shows the output of a MCMC code run on simulated data that contained 17 frequencies. The code infers that there are at least 16 frequencies, and possibly up to about 20 , with 16 the most probable solution. A typical output of the MCMC code used in this paper is shown in Figure 1 for the simulated data discussed in Section 3. After a short initial burn-in period, the distribution of the number of frequencies settles into the posterior distribution. The number of frequencies fluctuates between approximately 50 and 150, averaging around 90 . The actual number used was 61. Although our simulated data is different to that of Brewer et al. (2007), it does appear that the uniform prior results in additional frequencies being accepted than with the descriptive, regularly peaked prior, as could be expected. Since we do expect there to be significant departures from an equal spacing in some stars, we are prepared to accept the increased chance of detecting noise peaks and aliases between equally spaced frequencies for this comparison with Fourier methods. 

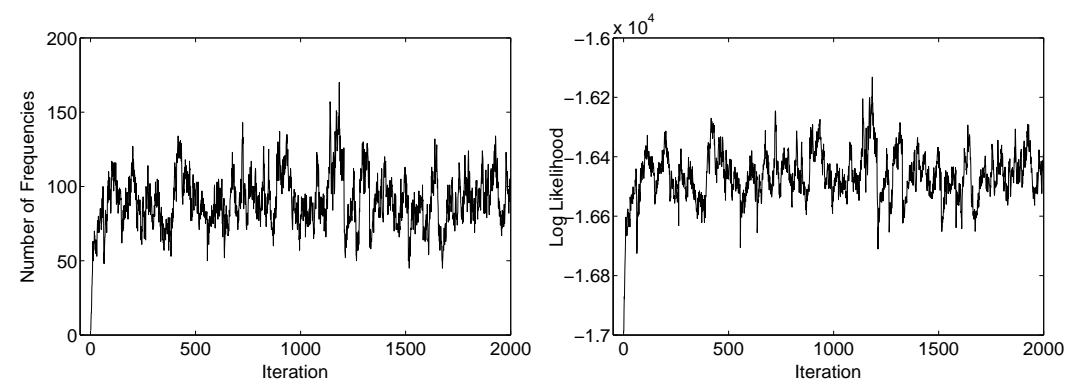

Figure 1: Results of a MCMC run with a uniform prior. Left Number of frequencies fitted as a function of iteration. Right Log likelihood of the proposed model as a function of iteration.

The original code, in comparison to traditional methods, had the deficiency of not returning the amplitudes of the modes, but only their relative probabilities. This has since been rectified, with the Markov Chain now sampling the distribution of both frequencies and amplitudes at each iteration.

\section{Tests with coherent oscillations}

Our first test of the algorithms was the simplest case possible, in which the oscillations are pure sinusoids and are well-separated in frequency. While no stellar oscillation is entirely coherent, there exist many cases where the mode lifetime is long enough for this to be a good approximation. For this test it was important to probe a variety of signal-to-noise $(S / N)$ ratios to gauge the performances of the programs at different levels. $\mathrm{S} / \mathrm{N}$ ratios from 2 and 5 were probed in this test.

The time series was generated as a sum of 61 sinusoids with frequencies separated by $60 \mu \mathrm{Hz}$, ranging from $300 \mu \mathrm{Hz}$ to $3900 \mu \mathrm{Hz}$. Phases were chosen at random. The generated signal was sampled every 100 seconds for a total of 9 days. Gaps are common in astronomical datasets, primarily due to the usual restriction of observations to be taken at night, which causes the aliasing that is so problematic to frequency analysis. To simulate this, only the first 40 per cent of a 'day' of simulated observations was retained as part of the time series. The resulting time series had 3114 data points. Random Gaussian noise with a standard deviation of $3.09 \mathrm{~ms}^{-1}$ was added to the data, which was calculated to provide an average noise in the amplitude spectrum of $0.10 \mathrm{~ms}^{-1}$ (from equations $A 1$ and $A 2$ of Kjeldsen \& Bedding 1995). The amplitudes chosen for the sinusoids varied linearly from $0.2 \mathrm{~ms}^{-1}$ at $300 \mu \mathrm{Hz}$ up to $0.5 \mathrm{~m}^{-1}$ at 

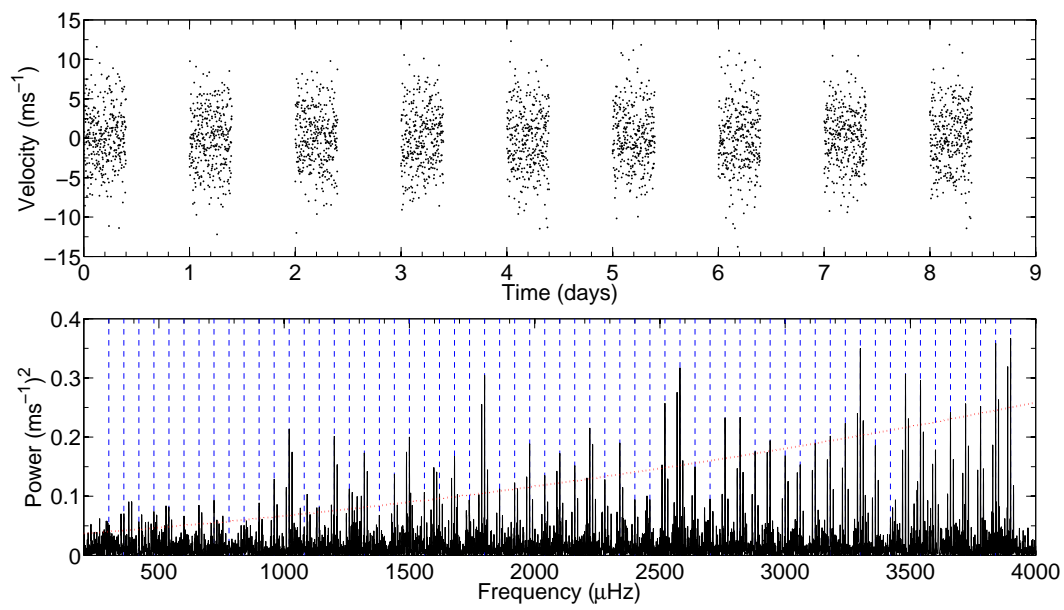

Figure 2: Top Simulated time series with coherent oscillations ranging in S/N from 2 to 5 . Bottom Corresponding power spectrum. Input frequencies are indicated by the vertical blue dashed lines. Input power as a function of frequency is indicated by the red dotted line. Sidelobes at $\pm 11.6 \mu \mathrm{Hz}$ due to daily gaps are clearly visible. Colour available on online version.

$3900 \mu \mathrm{Hz}$, to give the desired range of $\mathrm{S} / \mathrm{N}$ ratios. It should be noted that, due to the effects of noise, the measured $S / N$ of these peaks will differ from the input values, with some peaks being suppressed and others enhanced. The final time series is shown in Figure 2, together with its power spectrum. It is apparent that many more peaks are present in the power spectrum than the 61 input sinusoids marked by dashed lines, with some due to aliasing occurring at $\pm 11.6 \mu \mathrm{Hz}$ ( \pm 1 cycle/day) from the input peaks and others due to noise.

\subsection{Results}

The simulated time series was analysed with both versions of the iterative sine-wave fitting program, and with the Bayesian MCMC code. The peaks extracted by the fast iterative sine-wave fitting program and the probability spectrum output by the MCMC code are shown in Figure 3. It is immediately apparent from these graphs that both methods successfully identified the modes with the highest $\mathrm{S} / \mathrm{N}$ and that they agree in general. Although the relative heights of peaks are different between the two spectra in Figure 3, the modes with the highest $\mathrm{S} / \mathrm{N}$ generally correspond to those with the greatest posterior probability. 


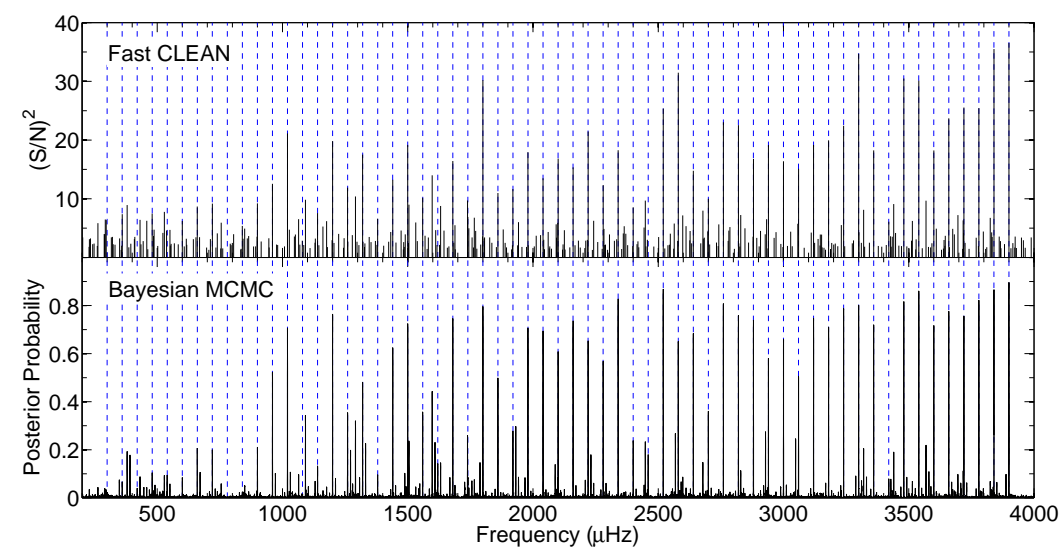

Figure 3: Top Peaks extracted by the fast iterative sine-wave fitting program. Bottom Probability spectrum from the Bayesian MCMC program. Blue dashed lines indicate the input frequencies.

To further see this relation between probability and S/N, Figure 4 compares the probability and $\mathrm{S} / \mathrm{N}$ of matched peaks between the Bayesian and Fourier methods. Between a $\mathrm{S} / \mathrm{N}$ of 2 and 4 there is a roughly linear relationship between $\mathrm{S} / \mathrm{N}$ and posterior probability. Either side of this region the probability saturates, resulting in a flattening of the graph. From this figure it is apparent that peaks can generally be believed (open circles) if the $S / N$ is above $\sim 3.5$ or the posterior probability is above $\sim 0.4$.

If a peak was identified by one program only, whether real, alias or noise, it appears on this graph on the vertical line of points at a $S / N$ of $\sim 1.1$ or the horizontal line of points at a posterior probability of $\sim 0.01$. If the iterative sine-wave fitting program did not find a peak above the $S / N \sim 1.1$ threshold it is either due to the peak having an inherently low $\mathrm{S} / \mathrm{N}$, or to the peak being diminished when a sine-wave was removed from the time series in previous iterations. This second explanation is responsible for the number of alias peaks in this category, which would be effectively removed in the Fourier case if the correct peak were identified first. However, if it happened that the fast Fourier program mistakenly identified the alias of a given peak, then it would not later detect the correct peak. The slow Fourier program would have a chance to change its identification, though in practice, this turned out to be rare. We found that the Bayesian program is more likely to identify both, and so may be of use as an alert to the possibility that a peak has been misidentified with 


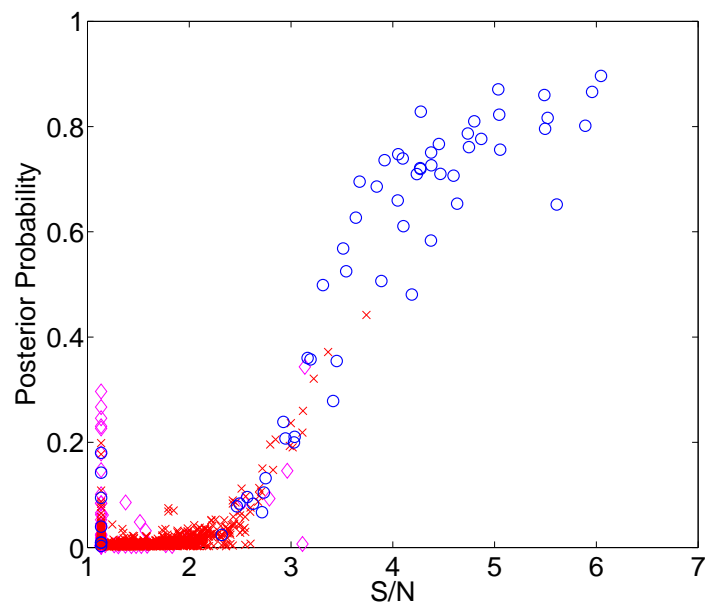

Figure 4: Posterior probability from Bayesian MCMC versus S/N from fast iterative sine-wave fitting program. Blue circles correspond to true input frequencies, magenta diamonds are aliases at $\pm 11.6 \mu \mathrm{Hz}$, and red crosses are noise peaks.

its alias. Comparing the échelle diagrams of the fast iterative sine-wave fitting program with the MCMC Bayesian code in Figure 5, we see that they effectively do equally well at identifying the correct peaks.

Despite the much longer time required to run the slow version of the iterative sine-wave fitting program, there did not appear to be any significant difference to the fast version, as can be seen in Figure 5. In general, the slow version is slightly more accurate in determining the frequencies, although in one case, the slow version has wrongly chosen the first and second aliases of the correct peak when the fast version did not. Stello et al. (2006) similarly found that the difference between the two was minor. They found that the fast version identified $1 \%$ false peaks compared to a perfect result by the slow version for simulated data from 100 time series with 17 equally-spaced frequencies with no noise, extracting 10 frequencies per time series. The scatter of the output frequencies relative to the input was roughly equal to the frequency resolution for the slow version, and about twice that size for the fast version. For noncoherent oscillations, the differences were diminished. The slow version takes a factor of $1.5(N+1)$ longer than the fast version, where $N$ is the number of frequencies to be extracted (Stello et al. 2006) and so, for a large number of frequencies, is prohibitively slow. 


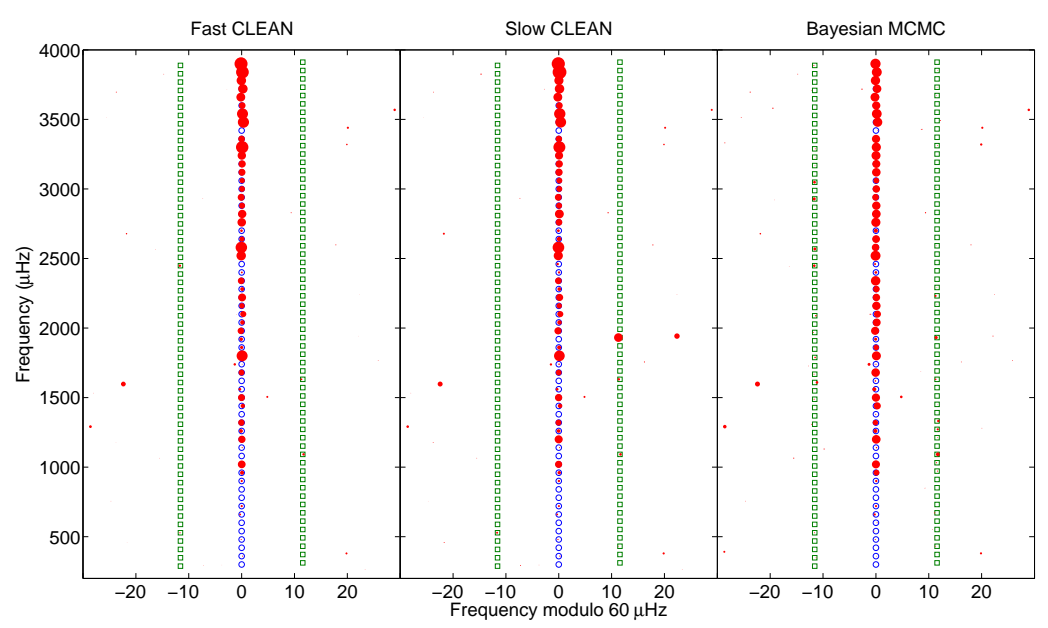

Figure 5: Left Échelle diagram from fast iterative sine-wave fitting program. Centre Échelle diagram from slow iterative sine-wave fitting program. Right Échelle diagram from Bayesian MCMC. The blue open circles indicate the location of real input peaks, with the green open squares indicating their aliases. Red filled circles indicate the peaks found by each program, with the size indicating the relative strength of each peak. The sizes have been scaled, so that peaks that follow the linear trend in Figure 4 will have approximately the same size in each diagram.

\section{Tests with problematic aliasing}

The next test investigates situations where aliasing is particularly problematic, that is, when the frequency separation between modes is close to an integer multiple of one cycle/day. The first side-lobes of adjacent peaks will coincide when the separation is exactly 2 cycles/day. A chirp was introduced to the input frequencies so that the frequency separation increased from 1.8 cycles/day up to 2.2 cycles/day, at a rate of 0.1 cycles/day/order. The input $\mathrm{S} / \mathrm{N}$ of each peak was fixed to 3.5. As previously mentioned, the effects of noise will modulate this in the output time series.

The window function and noise was the same as in the previous test (Section 3 ). The power spectrum of this time series is shown in Figure 6. The aliases are clearly apparent, approximately halfway between the real input peaks (dashed lines). Many of the sidelobes are higher than real peaks. 


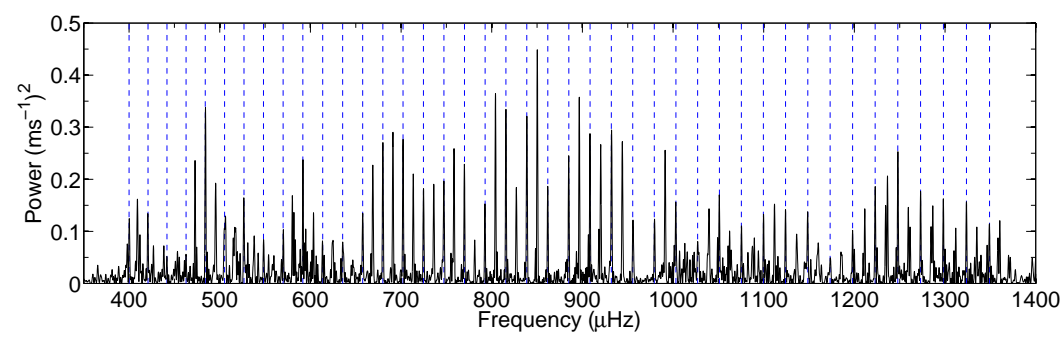

Figure 6: Power spectrum of simulated time series with problematic aliasing. Input frequencies are indicated by the blue dashed lines.

\subsection{Results}

Both programs performed quite well when the separation was significantly different from two cycles/day. When the aliases of adjacent modes coincide (in the middle of Figure 6), both failed to detect the correct frequencies. Matching the frequencies extracted by each program and plotting posterior probabilities against $\mathrm{S} / \mathrm{N}$ in Figure 7 , we see the same general trend as shown in Figure 4, although there is clearly more scatter. This scatter is due to the Bayesian MCMC code alternatively sampling both the real and alias peaks, and down- weighting their relative probabilities, whereas the iterative sine-wave fitting code will identify the highest peak at its $\mathrm{S} / \mathrm{N}$, real or alias, and miss the other altogether. This explanation is further borne out by the échelle diagrams, shown in Figure 8. The Bayesian method does have an advantage in drawing attention to the possibility of confusion, but is not any better at determining which is real.

\section{Discussion and Conclusions}

The Bayesian program and the traditional Fourier methods do equally well at identifying the correct frequency of stellar oscillations. The Bayesian method is effectively attempting to fit multiple sinusoids to the time series, whereas the iterative sine-wave fitting program finds the highest peaks, one at a time, from the Fourier amplitude spectrum. This often leads the Bayesian program to sample both the real peak and its aliases, whereas the Fourier program will identify only the highest of these. Without a more descriptive prior probability, it is not possible for the Bayesian program to avoid identifying alias or noise peaks that have high $\mathrm{S} / \mathrm{N}$. However, a more detailed prior might cause the results to be dominated by what was expected and not by what is actually present. It may be the case that the frequencies that are unexpected prove to be the most interesting. 


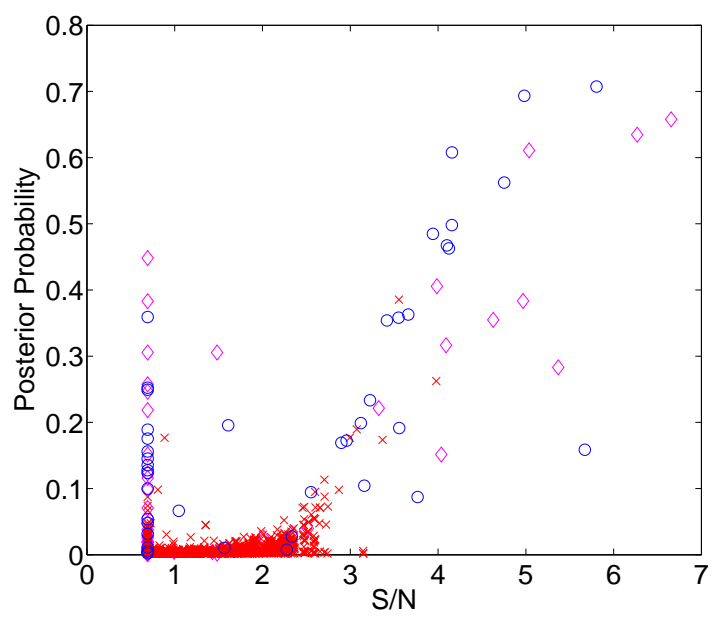

Figure 7: Same as for Figure 4 but for a time series with problematic aliasing.
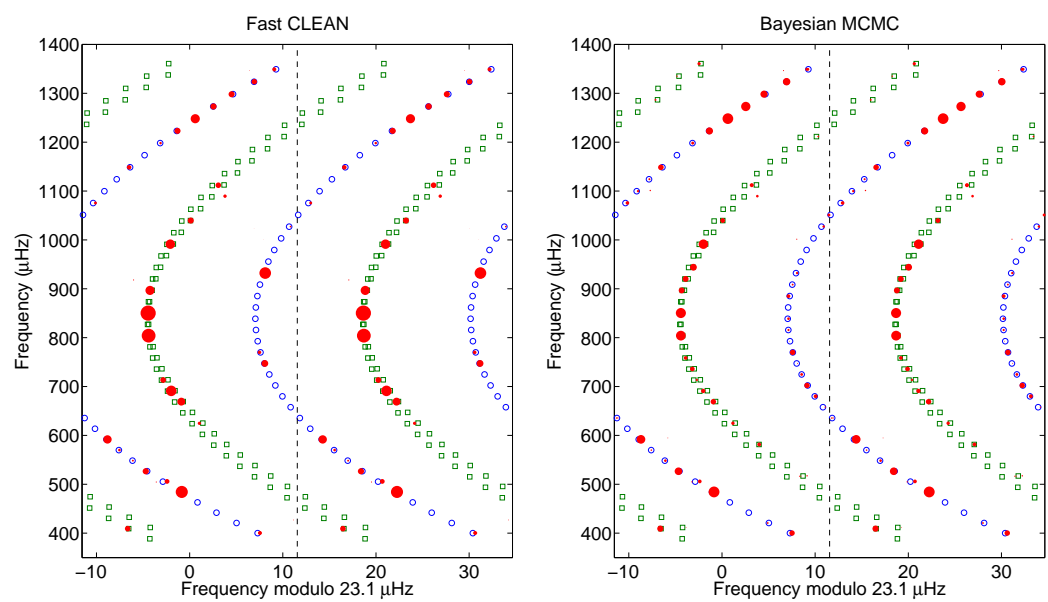

Figure 8: Échelle diagrams for a time series with problematic aliasing. Left Fast iterative sine-wave fitting, right Bayesian MCMC. Symbols same as for Figure 5. Note that the échelle diagrams have been plotted twice so that a ridge of input frequencies is continuous, with the dashed line indicating the half-way join. The ridge of input frequencies, and their aliases are curved due to the varying separation of the input series. 
When aliases are strong, the Bayesian method does have an advantage in highlighting the possibility of the confusion because it should detect both aliases and real peaks. In general however, we have found no advantage of the Bayesian method over the traditional Fourier methods. It is therefore recommended that the least computationally intensive program be used. The fast Fourier program was found to be fastest, provided there were not many peaks to be extracted, and there are a large number data points $(>\sim 10000)$. If a large number of peaks is required, and there are not too many data points, then the Bayesian method takes a comparable time. The slow Fourier program is prohibitively slow for determining any more than a few frequencies.

While we have shown that there is no major advantage in using the Bayesian approach discussed here, it is clear that Bayesian methods will continue to be used for fitting to the power spectrum once it has been calculated using traditional Fourier methods.

Acknowledgments. We acknowledge support from the Australian Research Council. TRW is supported by an Australian Postgraduate Award, a University of Sydney Merit Award and a Denison Merit Award.

\section{References}

Appourchaux, T. 2008, Astronomische Nachrichten, 329, 485

Bedding, T. R., Butler, R. P., Carrier, F., et al. 2006, ApJ, 647, 558

Bedding, T. R., Kjeldsen, H., Arentoft, T., et al. 2007, ApJ, 663, 1315

Benomar, O. 2008, Communications in Asteroseismology, 157, 98

Benomar, O., Appourchaux, T., \& Baudin, F. 2009, A\&A, 506, 15

Bretthorst, G. L. 1988, Lecture Notes in Statistics, Vol. 48, Bayesian Spectrum Analysis and Parameter Estimation (Springer-Verlag, New York)

Brewer, B. J., Bedding, T. R., Kjeldsen, H., \& Stello, D. 2007, ApJ, 654, 551

Brewer, B. J. \& Stello, D. 2009, MNRAS, 395, 2226

Carrier, F. \& Bourban, G. 2003, A\&A, 406, L23

Carrier, F., Kjeldsen, H., Bedding, T. R., et al. 2007, A\&A, 470, 1059

Gaulme, P., Appourchaux, T., \& Boumier, P. 2009, A\&A, 506, 7

Gregory, P. C. 2005, Bayesian Logical Data Analysis for the Physical Sciences: A Comparative Approach with 'Mathematica' Support (Cambridge University Press)

Kjeldsen, H. \& Bedding, T. R. 1995, A\&A, 293, 87

Kjeldsen, H., Bedding, T. R., Butler, R. P., et al. 2005, ApJ, 635, 1281 
Neal, R. M. 1993, Probabilistic Inference Using Markov Chain Monte Carlo Methods, Technical Report CRG-TR-93-1, Dept. of Computer Science, University of Toronto, available at http://www.cs.toronto. edu/ radford/

Roberts, D. H., Lehar, J., \& Dreher, J. W. 1987, AJ, 93, 968

Stello, D., Kjeldsen, H., Bedding, T. R., \& Buzasi, D. 2006, A\&A, 448, 709

Tassoul, M. 1980, ApJS, 43, 469

Tassoul, M. 1990, ApJ, 358, 313 
